TP Periodica Polytechnica Electrical Engineering and Computer Science

61(1), pp. 54-61, 2017

DOI: $10.3311 /$ PPee. 9777

Creative Commons Attribution (i)

RESEARCH ARTICLE

\section{Possibilities for On-line Detector Connectivity Checks in Beam Loss Monitoring Systems}

\author{
Csaba F. Hajdu ${ }^{1,2 *}$, Tamás Dabóczi ${ }^{2}$, Christos Zamantzas ${ }^{1}$
}

Received 20 July 2016; accepted after revision 30 October 2016

\begin{abstract}
Beam loss monitoring (BLM) is a method widely used for machine setup and tuning at particle accelerators, e.g. in physics research and medical applications. Additionally, the higher the energy and intensity of the particle beams, the more important the role of the BLM system in machine protection, which becomes critical for machines employing superconducting devices. A continuous and comprehensive surveillance of the entire BLM signal chain, in particular the functionality and connectivity of the detectors, is therefore essential. Nevertheless, no particle accelerator has this feature at present. This paper explores the options for implementing such a process for a new BLM system currently in development at the European Organisation for Nuclear Research (CERN).
\end{abstract}

\section{Keywords}

Beam loss monitoring (BLM), Connectivity check, On-line supervision

\section{Introduction}

Beam loss monitoring (BLM) involves measuring the flux of shower particles generated by the primaries lost from the beam and escaping the beam lines in a particle accelerator. The measurement data, acquired by detectors installed at strategically chosen locations throughout the accelerator such as machine aperture limits, may then be used for several purposes depending on the requirements of the accelerator.

Particle beams with high energy and intensity (number of particles) have a high damage potential. If required for protecting a machine from deterioration induced by such beams, the BLM system can trigger the safe extraction of the already circulating particles and the inhibition of any further injections. This is especially relevant for accelerators with superconducting devices, where a temperature rise caused by excessive energy deposition may have disastrous consequences, both in terms of repair cost and resulting machine downtime.

Moreover, the BLM data may be used to elaborate longterm strategies for limiting the activation of the machine caused by the beam. By minimizing the radioactivity induced in the components of the machine and the surrounding air, the cooldown time required between the shutdown and any human interventions on the machine can be reduced. This also allows lessening the radioactive risk members of staff operating on the machines are exposed to.

Additionally, subsets of the processed BLM data may be displayed in control rooms to assist the operators and archived for subsequent machine calibration and tuning.

A continuous and comprehensive supervision of the entire BLM signal and processing chain is therefore essential, yet to our knowledge, no particle accelerator in the world has this feature at present. This paper investigates the possibilities of implementing such a procedure in the context of a particular BLM system.

The rest of the Introduction deals with beam loss monitoring at the European Organisation for Nuclear Research (CERN), where the present work is being carried out. Section 2 presents the particular BLM system targeted by the supervision process, then Section 3 reviews the approaches that have been considered. The paper is concluded in Section 4. 


\subsection{The CERN accelerator complex in a few words}

CERN is among the world's leading particle physics research centers. It's home to a complex of particle accelerators dedicated to fundamental research. Before getting injected into the flagship particle collider and highest energy machine of the complex, the Large Hadron Collider (LHC), the energy of the particles is increased in stages through a series of smaller, lowenergy accelerators, usually referred to as the LHC injectors.

The LHC Injectors Upgrade (LIU) project mandates the consolidation of the aging injectors, including the overhaul of several machine protection systems. These machines must respond to new, more stringent constraints in order to increase beam brightness in the LHC.

\subsection{Beam loss monitoring at CERN}

BLM systems are the cornerstone of the strategy for machine protection and beam setup at CERN. The detectors in the BLM system of the LHC, and at hadron accelerators in general, are mainly ionization chambers [1]. Fig. 1 shows such a detector. The active volume of these detectors is filled with a gas, which is ionized by incident particle showers. A bias high voltage applied to the chamber separates the resulting electrons and ions, which are then collected on a stack of electrodes. The resulting current signal, carrying a charge proportional to the energy deposited in the detector by the ionizing particles, is acquired and digitized by the front-end cards of the system. The digitized output is transmitted to the back-end cards, which calculate several moving window integrals of various durations for every channel. These running sums are then compared to the corresponding beam abort thresholds to determine whether the initiation of a beam dump is necessary.

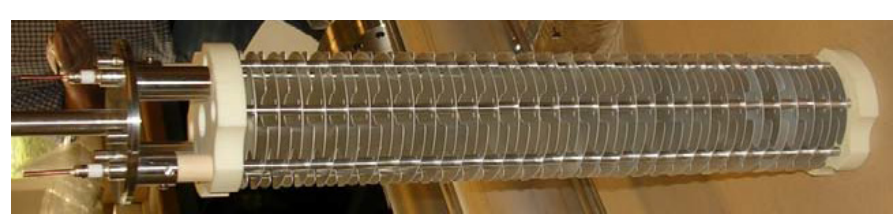

Fig. 1 An ionization chamber without its outer shell [2]. Notice the stack of parallel electrodes collecting the charges separated by the bias voltage.

In order to meet the exacting specifications in terms of failsafety and availability, various additional processes monitoring the status of the system have also been put in place in the LHC BLM system [3].

The requirements of the LIU project include the development of a new BLM system, common to all injectors. Since the characteristics of the accelerators vary widely, the acquisition frequency and input dynamic range of the new system need to be superior to its predecessors, and it also accommodates more varied detector types [4].

\subsection{Detector connectivity checks}

Currently, the LHC features the most comprehensive solution for supervising the connectivity of the BLM detectors and the functionality of the whole BLM system. This method, executable only while the accelerator is not in operation, enforces a connectivity check of each detector channel every 24 hours. It relies on inducing a sinusoidal modulation with a frequency of about $50 \mathrm{mHz}$ and an amplitude of about $30 \mathrm{~V}$ in the bias high voltage of the detectors, then measuring the resulting modulation in the digitized output current. The correct operation of the entire signal chain is a prerequisite for the presence of the modulation at the output of the corresponding detector. In case of a defective cabling connection, the modulation will be absent. Moreover, variations in the amplitude and the phase of the modulation in the output signal have been associated to various deteriorations in the signal chain, therefore, this process can also be used to survey the integrity of the components $[5,6]$.

An improved process must be elaborated, implemented, tested and integrated into the new BLM system for the injectors. The process should ensure a continuous functional supervision of the entire signal chain, from the detectors to the backend electronics.

\section{Beam loss monitoring at the injectors}

The accelerators of the injector complex are currently equipped with legacy BLM systems. At present, as imposed by the LIU project, the new BLM system intended to replace them is at an advanced stage of development. A prototype of the new system is installed in the laboratory and the first real installation operates at the Proton Synchrotron Booster (PSB) accelerator alongside the operational system. Another instance of the system is currently being installed at the Linac 4 accelerator, itself under installation and commissioning at the time of writing.

The new BLM system was designed with high performance and flexibility in mind, aiming to meet the demanding specifications for reliability and availability. Fig. 2 shows the signal acquisition and processing architecture of the system. Data processing relies on reconfigurable FPGA devices in order to ensure versatility and high data throughput, and to easily meet the varying requirements of the different accelerators. Contrary to the LHC system, all electronics will be installed in protected locations, thus radiation tolerance is not required.

\subsection{The analog signal chain}

In most locations throughout the injectors, ionization chambers identical to those used in the LHC will be installed as detectors for the new BLM systems. In order to keep them within the ionization operational range [1], a bias high voltage of about $1500 \mathrm{~V}$ is employed. This high voltage is provided by a pair of Heinzinger NCE 3000 series high voltage power supplies connected in parallel through serial diodes, installed in each 


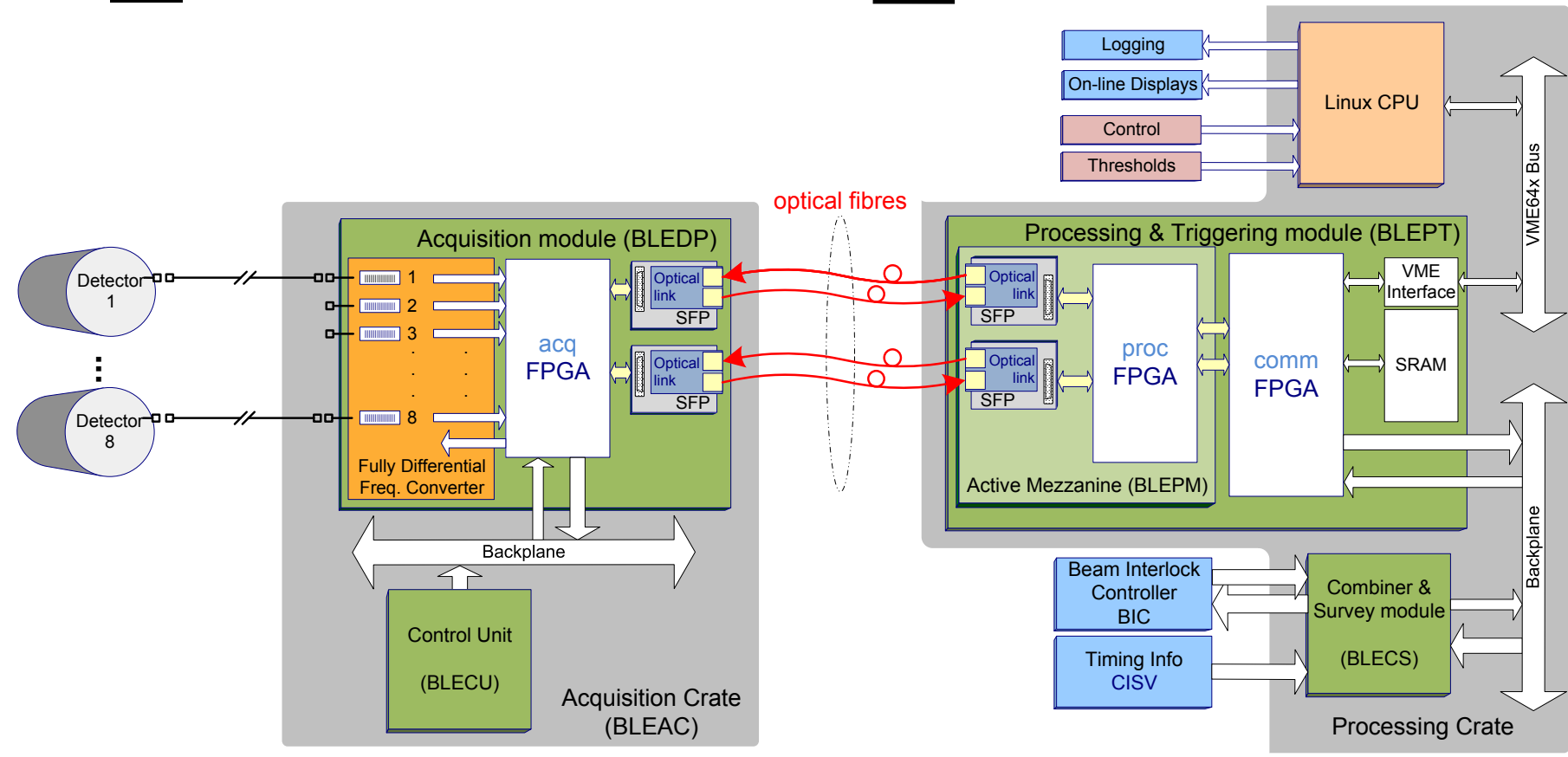

Fig. 2 Functional block diagram of the fully differential integrator [4].

acquisition rack. The output voltage of the power supplies can be adjusted between $0 \mathrm{~V}$ and $3000 \mathrm{~V}$ through an analog setpoint input, and their output current is limited to $20 \mathrm{~mA}$. This functionality is controlled by the BLECS module (see Section 2.3). Since the output voltage of the primary power supply is set about $50 \mathrm{~V}$ higher, the secondary power supply is idle in normal operation, while this configuration allows it to seamlessly take over powering the circuit in case the primary fails.

A high voltage distribution box provides connections for 64 detectors per rack. They are connected to the distribution box through high-voltage BNC cables of up to $200 \mathrm{~m}$ depending on the location. The shielding of the high voltage cables is cut at the detectors in order to avoid creating a ground loop through the shielding of the signal cable.

Each ionization chamber has a low-pass filter at its high voltage input. Since the output current of the power supply is limited, the capacitor of this low-pass filter supplies the electrical charge required if the detector sources high current due to an intense beam loss event. For each group of 4 channels, the high voltage distribution box features $1 \mathrm{M} \Omega$ serial resistors along with a resistor of $200 \mathrm{M} \Omega$ in parallel to the channels. This shunt allows a slow discharge of the filter capacitors in case the power is cut suddenly on the side of the power supply.

The output current of each detector is carried to the input of the electronics through custom-made triaxial signal cables for improved noise immunity. The length of these cables matches that of the corresponding high voltage cables.

We made a simulation model for part of the signal chain of a single detector channel. The results of the simulation are presented in Section 3.3 and the model itself is described in Appendix A.

\subsection{Front-end electronics}

Every acquisition rack hosts a front-end crate, which in turn accommodates up to eight front-end acquisition cards. Each of these cards has analog inputs for connecting eight detectors to an acquisition stage designed to be flexible and support various detector types. In order to stabilize the circuit against noise, a constant offset current is injected into every analog input in parallel to the signal under measurement. Along with ionization chambers, the use of other detector types such as diamond detectors, secondary emission monitors and Cherenkov monitors is foreseen in certain locations [4].

The output current of the detectors has a very high dynamic range. In order to digitize it, a novel measurement technique based on a fully differential integrator [7] was implemented on the front-end acquisition modules. It allows acquiring currents between $10 \mathrm{pA}$ and $200 \mathrm{~mA}$. This range is covered by two different operational modes implemented by two separate circuits. At the upper end of the dynamic range, the current is converted into a voltage which is acquired directly by an ADC. Currents up to $100 \mu \mathrm{A}$, however, are always acquired by the fully differential integrator. The switching between the two modes is managed by the FPGA of the acquisition board, which is also responsible for transmitting the digitized values generated with a sampling rate of $500 \mathrm{kHz}$ to the back-end card for processing [8].

The fully differential integrator [8], shown in Fig. 3, features two integrator capacitors. At any given point in time, the input current is integrated on one of the capacitors, whose input point is at virtual ground, since the other input of the differential amplifier is grounded through the input switch. Meanwhile, the other capacitor is discharged at the same rate. When the voltage 
on the capacitor being charged crosses a threshold, the input switch flips and the capacitors change roles. At this moment, a voltage pulse referred to as count is generated. The frequency of the counts will be proportional to the average magnitude of the input current. In order to improve the resolution in current, the output voltage of the capacitors is sampled by an ADC, thus yielding partial counts. All this information is combined into a digitized current value by the FPGA of the acquisition card.

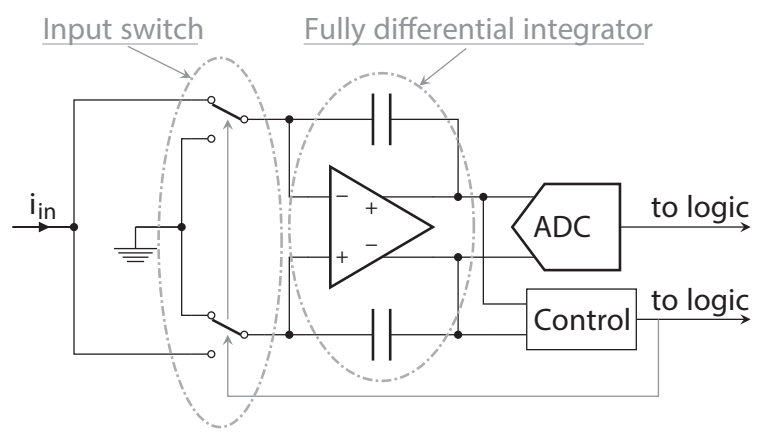

Fig. 3 Functional block diagram of the fully differential integrator [8].

\subsection{Back-end electronics}

A VME64x crate is also installed in every acquisition rack. It hosts up to eight back-end processing modules, a Linuxbased front end computer, accelerator timing receiver cards and a Combiner and Survey (BLECS) module.

The back-end cards receive the digitized data from the corresponding front-end over bidirectional optical links. The two FPGAs on these boards are responsible for calculating the running sums, comparing the values to the corresponding abort thresholds and assisting the decision concerning beam injection and circulation. They also publish the processed data and status information over the VME bus.

The BLECS card distributes accelerator timing and status signals throughout the VME crate. It also aggregates the beam dump requests coming from the processing cards and forwards them to the beam interlock system, and it supervises the system sanity check procedures [4]. Moreover, it also controls the high voltage power supplies through their analog setpoint inputs. Each of these lines is driven by the summed output of two 16-bit DACs. One of the DAC output voltages is scaled down through a voltage divider, which was foreseen to allow adding a modulation signal with a finer amplitude resolution to the coarser steady-state value.

\section{Connectivity checks in the new BLM system 3.1 Connectivity checks in the literature}

In the literature, various methods that can be interpreted as connectivity checks have been presented in a wide range of domains. In general, these methods involve measuring a transmission parameter of a two-port in some manner through its response to an excitation. Examples include the standard $4-20 \mathrm{~mA}$ measurement technique [9], the algorithmic minimization of the number of $k$-probe tests required for the connectivity verification of multi-chip module (MCM) substrates [10] and the quality control of through-silicon vias (TSV) in 3D integrated circuit technology [11]. Time domain reflectometry (TDR) [12] is employed to find fault locations in electrical wires [13] and optical fibers [14], and also for measuring the electrical properties of soil [15]. The moisture content of soils is also often measured using time-domain transmissometry (TDT) [16]. More broadly interpreted connectivity checking also occurs in contexts such as wireless sensor networks [17], power distribution networks [18], graph theory [19], linguistics [20], zoology [21] and medicine [22].

The detector connectivity checks in the BLM system of the LHC $[5,6]$ and the connectivity checks in the Transition Radiator Tracker (TRT) detector of the ATLAS experiment at CERN [23] are related methods from the field of particle accelerators. Less directly related topics from the same field include performance validation testing of the cathode strip chambers of the muon system of the CMS detector [24] and solder bump quality checking in the ATLAS pixel detector [25].

Our aim is the development of an on-line connectivity check for the new BLM system, with particular emphasis on the functionality of the analog part of the signal chain. Many methods cited above are not applicable to this measurement because they rely on techniques which are not readily adaptable to our application. In some cases, connectivity checking is only mentioned in passing and appears to be carried out by human intervention before installation or during commissioning, which is clearly unsuitable for our purpose. Since we are measuring a current signal and there is no DC continuity from the high voltage power supplies to the acquisition electronics, methods based on the standard 4-20 mA measurement technique are not applicable in our case, either. Since there is no readily available way of acquiring reflected waves at the high voltage power supplies, TDR also appears impractical. A quick attempt to assess detector connectivity through noise statistics inspired by the technique employed in the ATLAS TRT yielded discouraging results with our system.

The method presented in Section 3.3 draws inspiration from the detector connectivity checks in the BLM system of the LHC. The basic idea of this method can be interpreted as an adaptation of the TDT measurement principle.

\subsection{A non-invasive approach}

In order to assess the capabilities of the signal chain, an extensive series of measurements was executed with the prototype of the new BLM system [26]. The measurements showed that a prominent spectral component and several of its harmonics are consistently present in the digitized detector output current. The slowly varying frequency around $30 \mathrm{kHz}$ of this signal corresponds to the switching frequency of the high voltage power supply (HVPS). Detecting these components appeared 
very appealing for a connectivity check, since it might allow probing the integrity of the signal chain without any intervention on the analog side, by simply post-processing the digital signal in parallel to the operational signal processing.

In order to detect these frequency components, we implemented a structure based on the resonator-based spectral observer (Fourier analyzer, FA) proposed by Péceli [27], complemented by a frequency adaptation method based on that first suggested by Nagy [28]. As demonstrated in Fig. 4, the processing is capable of tracking the slow drift of the frequency satisfactorily. It also estimates the amplitudes of the peaks we are aiming to detect with reasonable precision.

However, additional measurements have revealed that the way the parasitic signal generated by the HVPS is coupled into the signal chain restricts the potential usability of this method to a limited number of cases, so it was necessary to search for an alternative method.

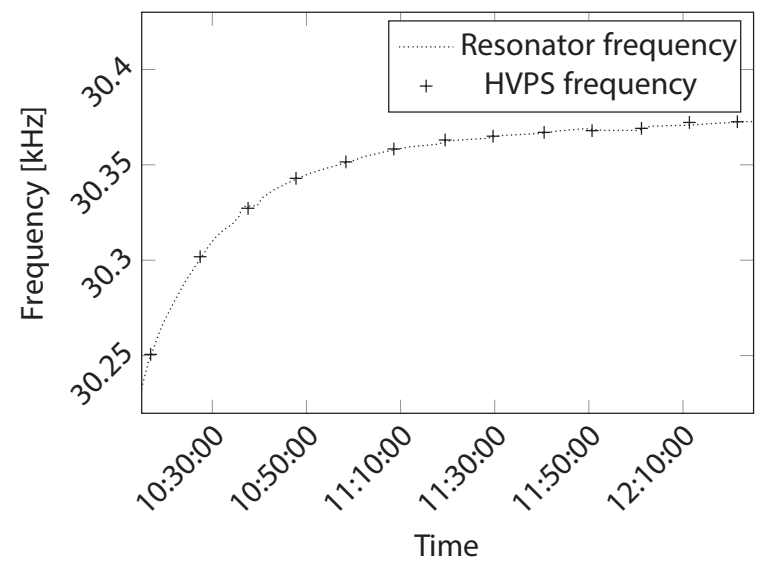

Fig. 4 Frequency tracking by the resonator-based observer with frequency adaptation: progression of the fundamental resonator frequency vs. variation of the fundamental frequency of the HVPS contributions. Every HVPS marker $(+)$ represents an acquisition.

\subsection{A modulation-based approach}

Giving up the potential advantages offered by a non-invasive method, we then considered an approach based on modulating the bias high voltage of the detectors, inspired by the process operating in the LHC. Fig. 5 illustrates the signal chain.

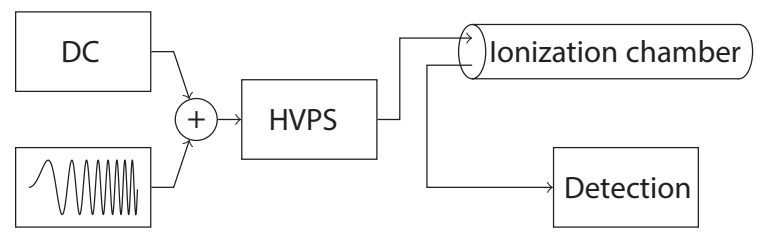

Fig. 5 Schematic view of the signal chain used for the modulation.

The high voltage power supplies of the BLM system are capable of producing a modulated output, even though they are not specified for AC voltages. Nevertheless, as revealed by the measurement campaign executed with the prototype system [26], certain limitations need to be taken into account. In the current configuration, the maximum modulation amplitude supported by the BLECS card is approximately $250 \mathrm{~V}_{\mathrm{PP}}$. With this amplitude, the power supplies produce a sine wave of good quality up to about $5 \mathrm{~Hz}$, but at higher frequencies, a non-linear distortion starts appearing. Upon further investigation, the probable cause is that the power supplies are only active when the output voltage increases. Any decrease in voltage appears to rely on the output capacitors of the power supply discharging through the internal resistance and the load impedance, which results in an exponential decay limiting the maximum rate of voltage decrease on the output. If this limitation is taken into consideration, the cutoff frequency at the output of the power supplies is about $30 \mathrm{~Hz}$.

We studied the frequency response beyond the power supplies using the simulation model presented in Appendix A. Our results, shown in Fig. 6, indicate that we should expect a significant decrease in the upper cutoff frequency of the system with increasing high voltage cable length. For the simulation, the cable lengths were chosen to agree with those typical in the laboratory system and in the PSB installation: $3 \mathrm{~m}$ and $40-110 \mathrm{~m}$, respectively.

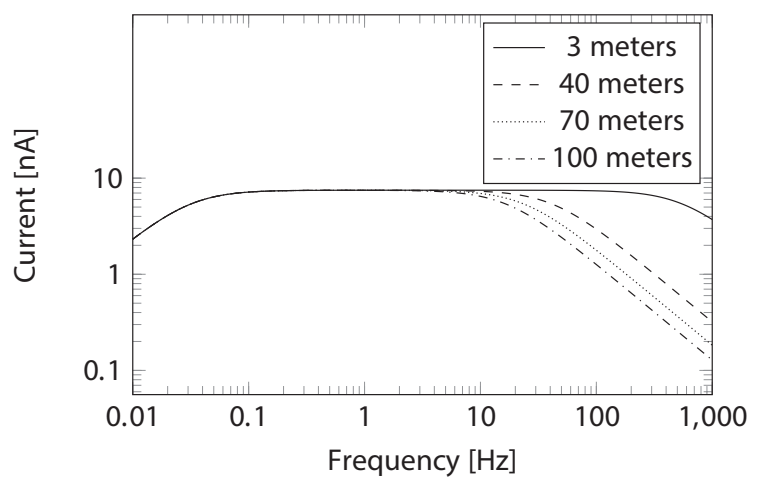

Fig. 6 Frequency response of the analog signal chain beyond the high voltage power supplies, as obtained by simulation for different cable lengths. The upper cutoff frequencies are $568 \mathrm{~Hz}, 42.7 \mathrm{~Hz}, 24.4 \mathrm{~Hz}$ and $17.1 \mathrm{~Hz}$ for $3 \mathrm{~m}, 40 \mathrm{~m}, 70 \mathrm{~m}$ and $100 \mathrm{~m}$, respectively.

For the injectors, a different modulation scheme is required than that used in the LHC, since the operation of the latter is marked by the recurrence of lengthy periods without beam, referred to as injection preparation. This means that there is time available to execute the connectivity checks requiring the machine to be out of operation, which take about 6 minutes in this implementation. On the other hand, the operation of the injectors is essentially uninterrupted, and we are aiming for continuous supervision. Therefore, it is a great advantage that the usable frequency range far exceeds that available in the LHC system. Even though the injectors operate continuously, this happens in pulsed mode. In the case of the PSB accelerator, the period of these pulses, referred to as a basic period of the machine, is $1.2 \mathrm{~s}$. In every basic period, there is about $0.5 \mathrm{~s}$ 
without beam. In this time slot, connectivity check measurements can be executed while avoiding any interference with the operational beam loss measurements.

According to our measurements, if care is taken to start and stop the modulation at the steady-state value of the high voltage, neither of these operations causes transients in current. By exploiting this fact, we conceived a scheme where the modulation is started after the beam has been extracted from the machine and it is stopped before the new injection. We implemented this procedure in the firmware of the BLECS module for testing. We chose a swept-frequency sine signal (chirp) for the modulation in order to ensure a more unique signature than with a conventional sinusoid. Fig. 7 shows acquisitions of the digitized detector output current made in the lab and PSB systems. Even though no effort has been made to ensure numerical consistency, the tendencies we observe in the measurements are in perfect agreement with the predictions of our simulation: the frequency response in the laboratory is dominated by the cutoff frequency of the high voltage power supplies, while in the PSB installation, the length of the high voltage cables starts to play a significant role.

In order to detect the response to the excitation in the acquired signal, we implemented a matched filtering process on one of the FPGAs of the backend processing module using this excitation as reference. This process calculates the cross-correlation of the reference signal $f$ to the incoming data stream $g$ to evaluate their similarity:

$$
(f \star g)[n]=\sum_{m=-\infty}^{\infty} f[m] g[m+n] .
$$

Since the reference waveform is of finite duration, $m$ is limited to a finite domain corresponding to the length of $f$. Additionally, the input data arrive as a stream, which makes the FPGA-based calculation of the cross-correlation straightforward. By storing the incoming samples of the signal stream in an appropriately sized circular buffer, then computing the inner product with the reference waveform whenever a new sample is received from the stream, we get a cross-correlation function value corresponding to an incremented $n$ upon the arrival of each new sample. According to our tests executed in MATLAB, detection would be possible using the raw data at $500 \mathrm{ksps}$, however, the required processing resources would not fit into the FPGA at hand. Instead, we decided to use one of the running sums already calculated on the back-end processing module, with a window length of $1 \mathrm{~ms}$. Then, in order to eliminate the influence resulting from beam losses, we apply windowing to the signal, setting all values to zero in the time period when beam is expected to be present. A typical crosscorrelation waveform obtained in the PSB is shown in Fig. 8. The non-linear distortions caused by the frequency-dependent response of the signal chain are limited and appear to have a negligible impact on detectability, even though for simplicity, we chose to use the excitation signal as reference for the detection by cross-correlation instead of filtered acquisitions from each detector channel.

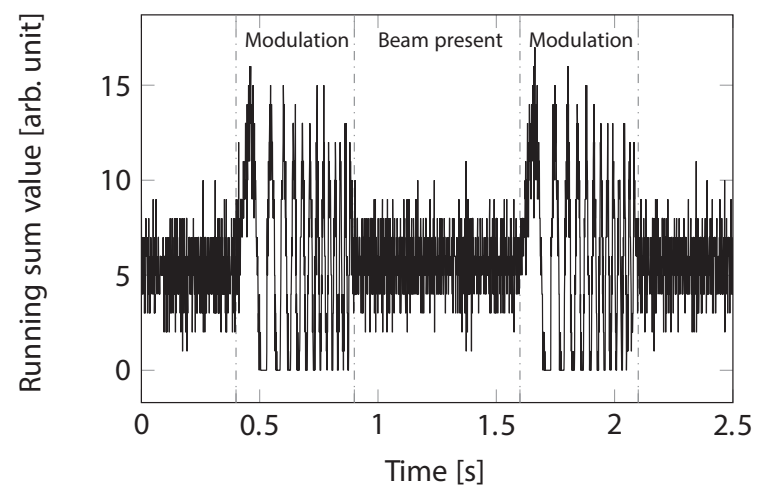

(a) Acquisition made in the laboratory, running sum window length: $1 \mathrm{~ms}$. Cable lengths: $3 \mathrm{~m}$. Frequency sweep: $0 \mathrm{~Hz}$ to $50 \mathrm{~Hz}$. The amplitude of the resulting current slightly exceeds that of the offset current, and since the system can only acquire positive input current, this yields the clipping visible in the signal at 0 .

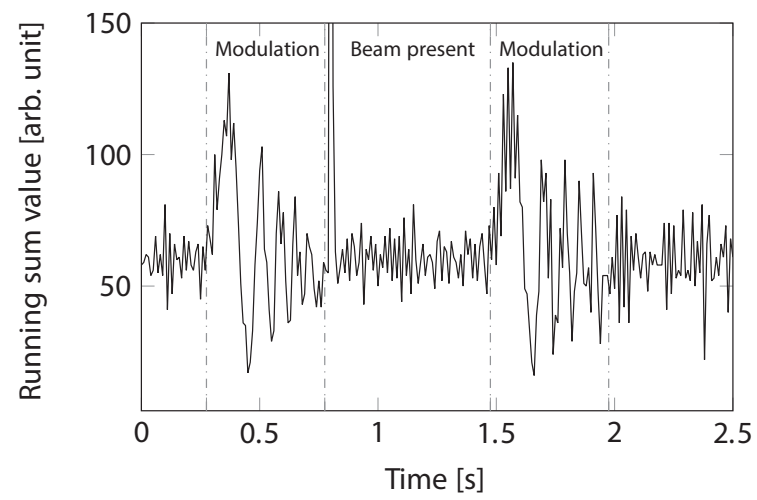

(b) Acquisition made with the PSB system, running sum window length: $10 \mathrm{~ms}$. Cable lengths: $110 \mathrm{~m}$. Frequency sweep: $0 \mathrm{~Hz}$ to $20 \mathrm{~Hz}$. Note the reduction in the amplitude of the modulation as the frequency increases. The magnitude of the offset current and the modulation are the same as in the previous acquisition. The tenfold increase in running sum values is due to the longer window length, so that the features of the signal remain distinguishable in this noisier environment. The clipped peak at the start of the "Beam present" interval is a beam loss.

Fig. 7 Digitized response to chopped chirp excitation in the lab and PSB systems. Duration of chirp: $500 \mathrm{~ms}$. Repetition period: $1.2 \mathrm{~s}$.

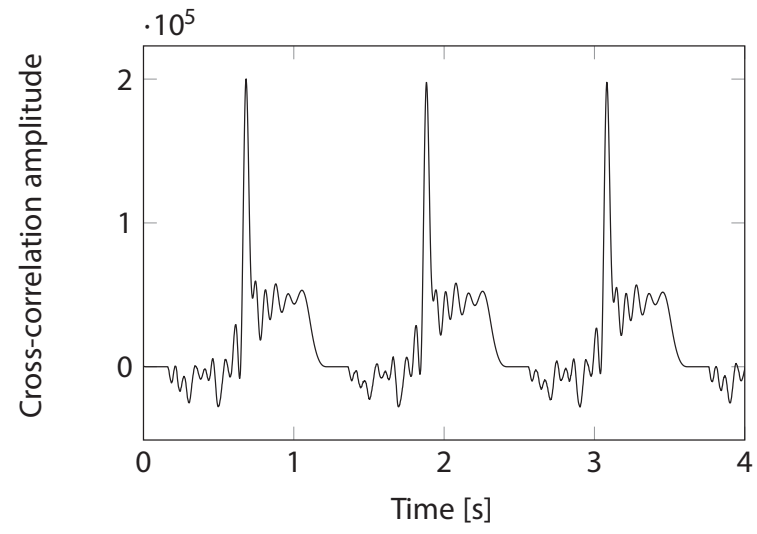

Fig. 8 Cross-correlation waveform obtained in the PSB using a $0-20 \mathrm{~Hz}$ swept sine excitation and corresponding matched filter at a sample rate of $1 \mathrm{ksps}$. The peaks promise excellent detectability. The shift in baseline is due to the windowing method used to eliminate beam loss contributions. Methods for its mitigation are being investigated at present. 


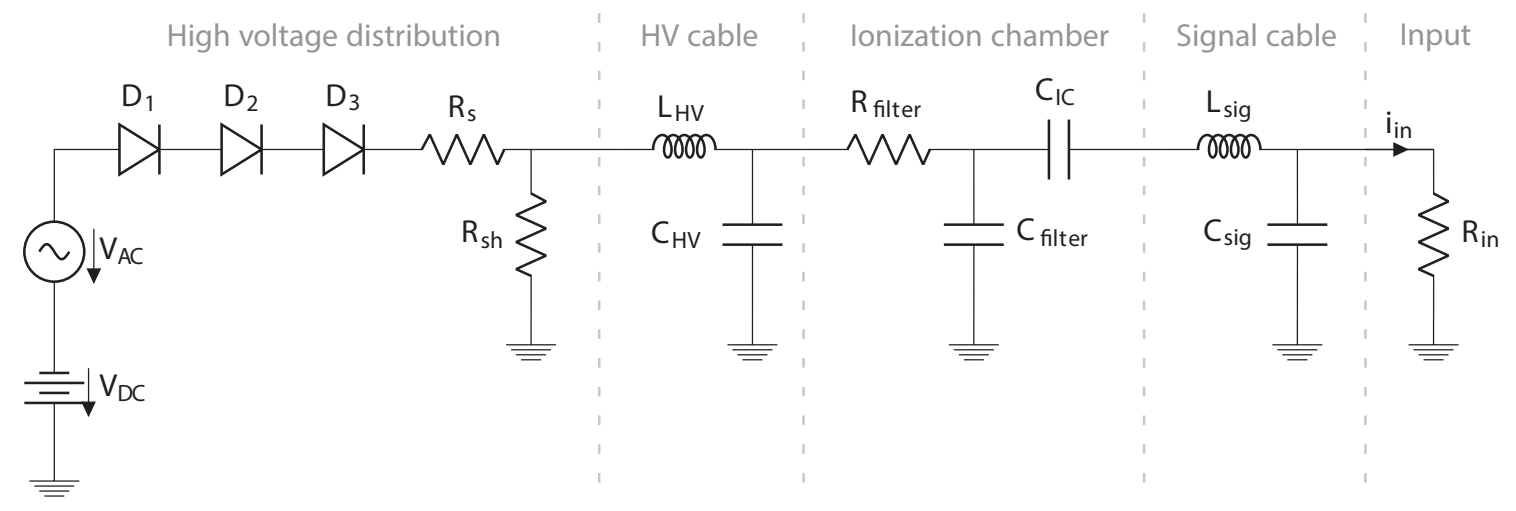

Fig. 9 Model of the analog signal chain beyond the high voltage power supplies used for simulation.

\section{Conclusions}

Along with the abandoned approach, we presented a feasible and promising way of implementing a connectivity check in the beam loss monitoring system under development for the injectors at CERN. We have a partially working implementation of the corresponding process.

The algorithm still needs some improvement and there is also room for fine-tuning. Moreover, we will need to ascertain whether this method is suitable for surveying the integrity of the components of the signal chain, similarly to the procedure used in the LHC BLM system.

\section{Appendix A The simulation model}

Our model for simulating the frequency response of the analog signal chain is shown in Fig. 9.

We decided not to attempt modeling the undisclosed internals of the real high voltage power supplies and used ideal voltage sources instead. In the high voltage distribution, $D_{1}$ and $D_{2}$ are 1N4007-type rectifier diodes, used for the parallel connection of the two power supplies as described in Section 2.1. $D_{3}$ is another 1N4007 diode, while $R_{s}$ is a serial resistor and $R_{s h}$ is the shunt resistor allowing a slow discharge of the input filters of the ionization chambers, placed ahead of each cluster of 4 chambers. $R_{s}=1 \mathrm{M} \Omega, R_{s h}=200 \mathrm{M} \Omega$.

The next stage in the model represents the Draka CBH50 type coaxial high voltage cable powering the chambers. According to the datasheet of the cable, the DC resistance of the conductors and the insulator capacitance can be ignored for our purposes. The mutual capacitance of the wires is $101 \mathrm{pF} / \mathrm{m}$, which was used for calculating the values of $C_{H V}$ corresponding to different cable lengths. We estimated the series inductance as $L / h=\frac{\mu_{0} \mu_{r}}{2 \pi} \ln D / d=0.2374 \mu \mathrm{H} / \mathrm{m}$ [29]. This, however, yields negligible values for $j \omega L_{H V}$ in our frequency range of interest, up to max. $1 \mathrm{kHz}$.

The ionization chamber was modeled using the component values of the RC filter at its input and the equivalent capacitance of a single ionization chamber [5]. $R_{\text {filter }}=10 \mathrm{M} \Omega$, $C_{\text {filter }}=470 \mathrm{nF}, C_{\text {monitor }}=312 \mathrm{pF}$.
The Draka CKC50 type coaxial signal cables were modeled based on considerations similar to those used for the high voltage cable. $C_{s i g} / h=82 \mathrm{pF} / \mathrm{m}, L_{s i g} / h=0.2285^{\mu \mathrm{H}} / \mathrm{m}$.

The analog front-end card has an input impedance of $50 \Omega$, thus we used this value for $R_{i n}$.

Since the analog front-end cards digitize a current, we are interested in the value of the system input current $i_{i n}$ in function of $V_{A C}$. In order to have a setup similar to the actual operating conditions, we set $V_{D C}=1500 \mathrm{~V}$ and $V_{A C}=125 \mathrm{~V}$.

As the impedance of $C_{I C}$ is three orders of magnitude higher than that of $C_{\text {fitter }}$ at all frequencies, we can predict that the part of the model from $C_{I C}$ to $R_{i n}$ will have little influence on the frequency domain behavior of the system. This part can be expected to mimic the behavior of the rest of the model, with currents reduced by three orders of magnitude. Then, since the impedance of $L_{H V}$ is negligibly small at the frequencies we are interested in, the frequency response of the system will be governed by the $\pi$ filter consisting of $C_{H V}, R_{\text {filter }}$ and $C_{\text {filter }}$. $C_{\text {filter }}$ will yield the high-pass behavior independent of cable length, while $C_{H V}$ causes the low-pass effect dependent on cable length.

\section{Acknowledgment}

Tamás Dabóczi acknowledges the support of ARTEMIS JU and the Hungarian National Research, Development and Innovation Fund in the frame of the R5-COP project.

\section{References}

[1] Zhukov, A. "Beam Loss Monitors (BLMs): Physics, Simulations and Applications in Accelerators." In: Proceedings of the 2010 Beam Instrumentation Workshop (BIW10)." Santa Fe, NM, USA, May 2010, pp. 553-565. URL: http://accelconf.web.cern.ch/Accelconf/BIW2010/ papers/thtnb01.pdf

[2] Dehning, B., Effinger, E., Emery, J., Ferioli, G., Guaglio, G., Holzer, E. B., Kramer, D., Ponce, L., Prieto, V., Stockner, M., Zamantzas, C. "The LHC Beam Loss Measurement System." In: Proceedings of the 22nd Particle Accelerator Conference (PAC07), Albuquerque, NM, USA, June 2007, pp. 4192-4194. URL: http://epaper.kek.jp/p07/PAPERS/FRPMN071.PDF 
[3] Zamantzas, C., Dehning, B., Effinger, E., Emery, J., Jackson, S. "RealTime System Supervision for the LHC Beam Loss Monitoring System at CERN." In: Proceedings of the 14th International Conference on Accelerator \& Large Experimental Physics Control Systems (ICALEPCS 2013), San Francisco, CA, USA, Oct. 2013, pp. 242-245. URL: http://accelconf. web.cern.ch/AccelConf/ICALEPCS2013/papers/moppc062.pdf

[4] Zamantzas, C., Alsdorf, M., Dehning, B., Jackson, S., Kwiatkowski, M., Viganò, W. "System Architecture for measuring and monitoring Beam Losses in the Injector Complex at CERN." In: Proceedings of the 1st International Beam Instrumentation Conference (IBIC2012), Tsukuba, Japan, Oct. 2012, pp. 347-350. URL: http://accelconf.web.cern.ch/AccelConf/IBIC2012/papers/tupa09.pdf

[5] Emery, J., Dehning, B., Effinger, E., Ferioli, G., Zamantzas, C., Ikeda, H., Verhagen, E. "LHC BLM Single Channel Connectivity Test using the Standard Installation." In: Proceedings of the 9th European Workshop on Beam Diagnostics and Instrumentation for Particle Accelerators (DIPAC09), Basel, Switzerland, May 2009, pp. 354-356. URL: http:// epaper.kek.jp/d09/papers/tupd26.pdf

[6] Emery, J., Dehning, B., Effinger, E., Nordt, A., Sapinski, M. G., Zamantzas, C. "First experiences with the LHC BLM sanity checks." Journal of Instrumentation. 5(12), C12044, 2010. https://doi.org/10.1088/1748-0221/5/12/C12044

[7] Viganò, W., Dehning, B., Effinger, E., Venturini, G. G., Zamantzas, C. "Comparison of three different concepts of high dynamic range and dependability optimised current measurement digitisers for beam loss systems." In: Proceedigs of the 1st International Beam Instrumentation Conference (IBIC2012), Tsukuba, Japan, Oct. 2012, pp. 66-70. URL: http://accelconf.web.cern.ch/AccelConf/IBIC2012/papers/mopa09.pdf

[8] Viganò, W., Alsdorf, M., Dehning, B., Kwiatkowski, M., Venturini, G. G., Zamantzas, C. "10 orders of magnitude current measurement digitisers for the CERN beam loss systems." Journal of Instrumentation. 9(2), C02011. 2014. https://doi.org/10.1088/1748-0221/9/02/C02011

[9] "Compatibility of analog signals for electronic industrial process instruments." Standard ANSI/ISA-50.1-1982 (R1992), 1992.

[10] Yao, S. Z., Chou, N. C., Cheng, C. K., Hu, T. C. "An optimal probe testing algorithm for the connectivity verification of MCM substrates." In: Proceedings of the 1992 IEEE/ACM International Conference on Computer-Aided Design, 1992 (ICCAD-92), Santa Clara, CA, Nov. 8-12, 1992, pp. 264-267. https://doi.org/10.1109/ICCAD.1992.279363

[11] Kim, J. J., Kim, H., Kim, S., Bae, B., Jung, D. H., Kong, S., Kim, J., Lee, J., Park, K. "Non-contact wafer-level TSV connectivity test methodology using magnetic coupling." In: Proceedings of the 2013 IEEE International 3D Systems Integration Conference (3DIC). San Francisco, CA, Oct. 2-4, 2013, pp. 1-4. https://doi.org/10.1109/3DIC.2013.6702328

[12] Cole, R. H. "Time domain reflectometry." Annual Review of Physical Chemistry. 28(1), pp. 283-300. 1977. https://doi.org/10.1146/annurev.pc.28.100177.001435

[13] Wang, A., Zhang, M., Xu, H., Wang, Y. "Location of wire faults using chaotic signal." IEEE Electron Device Letters. 32(3), pp. 372-374. 2011. https://doi.org/10.1109/LED.2010.2097237

[14] So, V., Lamont, J., Vella, P. "Fault-locating accuracy with an OTDR: reflective versus nonreflective faults." IEEE Journal on Selected Areas in Communications. 4(5), pp. 737-740. 1986. https://doi.org/10.1109/JSAC.1986.1146374

[15] Robinson, D. A., Jones, S. B., Wraith, J. M., Or, D., Friedman, S. P. "A review of advances in dielectric and electrical conductivity measurement in soils using time domain reflectometry." Vadose Zone Journal. 2(4), pp. 444-475. 2003. https://doi.org/10.2136/vzj2003.4440
[16] Will, B., Rolfes, I. "Comparative study of moisture measurements by time domain transmissometry." In: Proceedings of the IEEE SENSORS 2013, Baltimore, MD, Nov. 3-6, 2013, pp. 1-4. https://doi.org/10.1109/ICSENS.2013.6688529

[17] Shang, Y., Rumi, W., Zhang, Y., Fromherz, M. "Localization from connectivity in sensor networks." IEEE Transactions on Parallel and Distributed Systems. 15(11), pp. 961-974, 2004.

https://doi.org/10.1109/TPDS.2004.67

[18] Nahman, J. M., Perić, D. M. "Optimal planning of radial distribution networks by simulated annealing technique." IEEE Transactions on Power Systems. 23(2), pp. 790-795. 2008. https://doi.org/10.1109/TPWRS.2008.920047

[19] Even, S. "An algorithm for determining whether the connectivity of a graph is at least k." SIAM Journal on Computing. 4(3), pp. 393-396, 1975. https://doi.org/10.1137/0204034

[20] Kim, B., Lee, G. G., Lee, J-H. "Morpheme-based grapheme to phoneme conversion using phonetic patterns and morphophonemic connectivity information." ACM Transactions on Asian Language Information Processing (TALIP). 1(1), pp. 65-82. 2002. https://doi.org/10.1145/595576.595580

[21] Bowman, J., Fahrig, L. "Gap crossing by chipmunks: an experimental test of landscape connectivity." Canadian Journal of Zoology. 80(9), pp. 1556-1561, 2002. https://doi.org/10.1139/z02-161

[22] Bhuiyan, A., Nath, B., Chua, J., Ramamohanarao, K. "Automatic detection of vascular bifurcations and crossovers from color retinal fundus images." In: Proceedings of the Third International IEEE Conference on Signal-Image Technologies and Internet-Based System, 2007 (SITIS '07). Shanghai, China, Dec. 16-18, 2007. pp. 711-718.

https://doi.org/10.1109/SITIS.2007.86

[23] Ghodbane, N., Pons, X., Rohne, O. M. "A Method to check the Connectivity for the ATLAS TRT Detector." arXiv preprint physics/0510130, 2005. URL: https://arxiv.org/abs/physics/0510130

[24] Barashko, V. "Performance validation tests of the cathode strip chambers for CMS muon system." In: IEEE Nuclear Science Symposium Conference Record, 2005, Vol. 2, Fajardo, PR, USA, Oct. 23-29, 2005. pp. 827-829. https://doi.org/10.1109/NSSMIC.2005.1596383

[25] Gemme, C., Fiorello, A. M., Gagliardi, G., Gilchriese, M., Netchaeva, P., Rossi, L., Ruscino, E., Vernocchi, F., Varasi, M. "Study of indium bumps for the ATLAS pixel detector." Nuclear Instruments and Methods in Physics Research Section A: Accelerators, Spectrometers, Detectors and Associated Equipment. 465(1), pp. 200-203. 2001.

https://doi.org/10.1016/S0168-9002(01)00390-4

[26] Hajdu, C. F., Dabóczi, T., Zamantzas, C. "On-line system supervision of beam loss monitoring systems with sinusoidal excitation." In: $X X I$ IMEKO World Congress - Full Papers, Prague, Czech Republic, Sept. 2015. URL: https://www.imeko.org/publications/wc-2015/IMEKO-WC2015-TC10-237.pdf

[27] Péceli, G. "A common structure for recursive discrete transforms." IEEE Transactions on Circuits and Systems. 33(10), pp. 1035-1036. 1986. https://doi.org/10.1109/TCS.1986.1085844

[28] Nagy, F. "Measurement of signal parameters using nonlinear observers. IEEE Transactions on Instrumentation and Measurement. 41(1), pp. 152-155. 1992. https://doi.org/10.1109/19.126651

[29] Pozar, D. M. "Microwave Engineering." 4th ed., Chapter 2.2., John Wiley \& Sons, 2012. 